\title{
Statistical analysis of life-time and temperature of black holes
}

\author{
Ved Prakash ${ }^{1}$, Dipo Mahto ${ }^{2,}$,, Ashok Kumar ${ }^{3}$, Basant Kumar Das ${ }^{1}$ \\ ${ }^{1}$ University Department of Statistics \& Computer Application, T. M. B. U. Bhagalpur-812007, India \\ ${ }^{2}$ Department of Physics, Marwari College, T. M. B. U. Bhagalpur-812007, India \\ ${ }^{3}$ Department of Physics, M.R.J.D. College, Bishnupur, Begusarai-851101, India
}

\section{Email address:}

chandanbgp@gmail.com (V. Prakash),dipomahto@hotmail.com (D. Mahto),drashokbgs259@gmail.com (A. Kumar), krchandan@rediffmail.com(B. K. Das)

\section{To cite this article:}

Ved Prakash, Dipo Mahto, Ashok Kumar, Basant Kumar Das. Statistical Analysis of Life-Time and Temperature of Black Holes. American Journal of Theoretical and Applied Statistics. Vol. 2, No. 6, 2013, pp. 228-232. doi: 10.11648/j.ajtas.20130206.20

\begin{abstract}
In the present article, statistical analysis of lifetime and temperature of the black holes have been studied existing in X-ray binaries and active galactic nuclei with the help of formula $\Gamma=(\mathrm{M} / \mathrm{M} \odot) 3 \times 1066$ years and $\mathrm{T}=\mathrm{hc} 3 / 8 \pi \mathrm{kGM} \mathrm{Kelvin}$ respectively where $\mathrm{M}$ stands for the mass of black hole and other parameters have their usual meaning.
\end{abstract}

Keywords: Lifetime, XRBs, AGN, Supernova

\section{Introduction}

Like the living creatures; the star takes birth and finally dies. The black hole is the final stage of star formed after Supernova explosion. Due to this explosion the outer portion of the star is thrown out into the space leaving behind the remnant core [1]. This remaining core begins to contract due to its gravity and becomes infinite dense matter; the gravity of this dense matter becomes so high that nothing can escape from it. This dense matter formed after supernova explosion is called black hole. Classically black holes are perfect absorbers, but do not emit anything; their physical temperature is absolute zero [2]. But in 1974, Stephan Hawking showed that quantum field theory predicts that black holes should radiate energy like a black body with a temperature proportional to the surface gravity of the black hole [3]. Since the black hole radiates, it evaporates. Hence its lifetime is finite. Stephan Hawking provided a theoretical argument for its existence in 1974, and sometimes also after the physicist Jacob Bekenstein who predicted that black holes should have a finite, non-zero temperature and entropy [4]. Shunichiro Kinoshita and Norihiro Tanahashi discussed the Hawking temperature of near-equilibrium black holes using a semi-classical analysis and found that the temperature is determined by the surface gravity of the past horizon [5]. Triyanta \& Anike N. Bowaire concluded that in the case of the Schwarzschild black hole, a higher Hawking temperature corresponds to a lower mass of a black hole [6].
Mehta et al. derived an expression for the variation of temperature of the black holes with respect to mass using the temperature of black hole at which the black hole radiates to infinity all species of particles with a perfect black body spectrum and surface gravity for Schwarzschild black holes and they also calculated their values of different test black holes existing only in X-ray binaries [16].

In the present paper, we have studied the statistical analysis of the lifetime and temperature of the black holes and concluded that the life time of the black hole increases gradually with increase of the mass of different test black holes and the life time of the black hole decreases gradually with increase of the mass of different test black holes in XRBs, while like transverse wave pattern in AGN.

\section{Theoretical Discussion}

\subsection{Temperature of Black Hole}

A black hole is a Bose-Einstein ensemble of quanta of mass equal to twice the Planck mass, confined in a sphere of radius twice the black hole mass [9]. The concept of black hole entropy was introduced by Bekenstein which stated that it is proportional to the black hole area A. Later on, Bardeen, Carter and Hawking and Hawking performed calculations using a semi-classical approximation, putting Bekenstein conjecture on a firm basis. They established that the black hole temperature is proportional to its surface gravity [8]. On the other hand, A. Zee in his book: "Quantum Field Theory 
in a Nutshell", presents a nice intuitive derivation of the Hawking temperature and started from the Schwarzschild solution of Einstein equations for vacuum and finally obtained $[7,8]$ :

$$
T=\frac{1}{8 \pi M}
$$

Or,

$$
T=\frac{\hbar c^{3}}{8 \pi k G M}
$$

where $\hbar$ and c are Dirac-Plank constant and velocity of light respectively. These constants are restored in the right side of (2).

We know that

$$
\hbar=\frac{h}{2 \pi}
$$

Putting the value of eqn. (3) in eqn. (2) and solving, we obtain

$$
T=\frac{h c^{3}}{16 \pi^{2} k G M}
$$

Eqn. (4) is the required expression for the temperature of the black holes as proposed by Stephen Hawking in 1974.

\subsection{Life Time of Black Hole}

Now we have seen that a black hole "shines" with Hawking radiation. The escaping member of a virtual particle pair carried away energy from the black hole, and the black hole loses mass as a result. Eventually the black hole loses all its energy, or equivalently mass, and evaporates. The formula for the lifetime of a black hole is given by the following equation:

$$
\Gamma=\left(\mathrm{M} / \mathrm{M}_{\odot}\right)^{3} \times 10^{66} \text { years }
$$

Eqn. (5) represents the lifetime of black hole in terms of mass of the black hole and mass of the sun.

\section{Data in the Support of Black Holes}

There are two categories of black holes classified on the basis of their masses clearly very distinct from each other, with very different masses $\mathrm{M} \sim 5-20 \mathrm{M}_{\square}$ for stellar - mass black holes in X-ray binaries and $\mathrm{M} \sim 10^{6}-10^{9.5} \mathrm{M}_{\square}$ for super massive black holes in Active Galactic Nuclei [10].

Assuming an isotropic, spherically symmetric system, Sargent et al. detected a central dark mass $\sim 5 \times 10^{9} M_{\odot}$ within the inner $110 \mathrm{pc}$ of M87 [15]. A kinematical study of NGC4261 followed in 1996, claiming a $(4.9 \pm 1.0) \times 10^{8} M_{\odot}[13]$. Masses of "central dark object" have been estimated in about forty cases, using stellar dynamics, emission lines of orbiting gas and, most accurately, using water masers. They range from $\sim 2 \times 10^{6} M_{\odot} t o \sim 3 \times 10^{9} M_{\odot}$ and, in many cases, the compactness is sufficient to rule out star clusters with confidence [12]. The most viable scenario for modeling of active galactic nuclei includes a super massive black hole with the mass $10^{6}-10^{9} M_{\odot}$ accreting the galaxian matter from its vicinity [14]. Most detected SBHs are in the $10^{8} \leq M_{\bullet} \leq 10^{9} M_{\odot}$ range, there are no detections below $10^{6} M_{\odot}$ (the "building block" range) or above $10^{10} M_{\odot}$ (the brightest quasar range), and even the $10^{6} \leq M_{.} \leq 10^{7} M_{\odot}$ range is very poorly sampled [11].

On the basis of data mentioned above, we calculate the life time \& temperature of black holes in XRBs and in AGN for different test black holes listed in tables1 \&2 respectively.

\section{Data Used in Calculation}

$$
\mathrm{G}=6.673 \times 10^{-11} \mathrm{~m}^{3} \mathrm{~kg}^{-1} \mathrm{~s}^{-2}, \mathrm{~h}=6.623 \times 10^{-34} \mathrm{Js}, \quad \mathrm{k}=1.380 \times 10^{-23} \mathrm{~J} / \mathrm{K}, \mathrm{c}=3 \times 10^{8} \mathrm{~m} / \mathrm{s}, \mathrm{M}_{\odot}=1.99 \times 10^{30} \mathrm{~kg} .
$$

\begin{tabular}{|c|c|c|c|}
\hline S.N. & $\begin{array}{l}\text { Mass of black hole } \\
M_{\odot}=1.99 \times 10^{30} \mathrm{~kg}\end{array}$ & $\begin{array}{l}\text { Life time of black hole } \\
\Gamma=\left(M / M_{\odot}\right)^{3} \times 10^{66} \text { years }\end{array}$ & $\begin{array}{l}\text { Temperature of black hole } \\
T=h^{3} c / 8 \pi k G M(\text { kelvin })\end{array}$ \\
\hline 1 & $5 \mathrm{M}_{\square}$ & $1.25 \times 10^{68}$ & $7.8278 \times 10^{-8}$ \\
\hline 2 & $6 \mathrm{M}_{\square}$ & $2.16 \times 10^{68}$ & $6.5232 \times 10^{-8}$ \\
\hline 3 & $7 \mathrm{M}_{\square}$ & $3.43 \times 10^{68}$ & $5.5913 \times 10^{-8}$ \\
\hline 4 & $8 \mathrm{M}_{\square}$ & $5.12 \times 10^{68}$ & $4.8924 \times 10^{-8}$ \\
\hline 5 & $9 \mathrm{M}_{\square}$ & $7.29 \times 10^{68}$ & $4.3488 \times 10^{-8}$ \\
\hline 6 & $10 \mathrm{M}_{\square}$ & $10.00 \times 10^{68}$ & $3.9139 \times 10^{-8}$ \\
\hline 7 & $11 \mathrm{M}_{\square}$ & $13.30 \times 10^{68}$ & $3.5581 \times 10^{-8}$ \\
\hline
\end{tabular}

\section{Table}

In the tables $1 \& 2$, we have calculated life time (in year) and temperature (in kelvin) of black holes in XRBs and AGN using the formula $\Gamma=\left(M / M_{\odot}\right)^{3} \times 10^{66}$ years and $T=h^{3} c / 8 \pi k G M(k e l v i n)$ respectively.

Table 1. Life time \& Temperature of Black Holes in XRBs 


\begin{tabular}{|c|c|c|c|}
\hline S.N. & $\begin{array}{l}\text { Mass of black hole } \\
M_{\odot}=1.99 \times 10^{30} \mathrm{~kg}\end{array}$ & $\begin{array}{c}\text { Life time of black hole } \\
\Gamma=\left(M / M_{\odot}\right)^{3} \times 10^{66} \text { years }\end{array}$ & $\begin{array}{l}\text { Temperature of black hole } \\
T=h^{3} c / 8 \pi k G M(\text { kelvin })\end{array}$ \\
\hline 8 & $12 \mathrm{M}_{\square}$ & $17.76 \times 10^{68}$ & $3.2616 \times 10^{-8}$ \\
\hline 9 & $13 \mathrm{M}_{\square}$ & $21.97 \times 10^{68}$ & $3.0107 \times 10^{-8}$ \\
\hline 10 & $14 \mathrm{M}_{\square}$ & $27.40 \times 10^{68}$ & $2.7956 \times 10^{-8}$ \\
\hline 11 & $15 \mathrm{M}_{\square}$ & $33.75 \times 10^{68}$ & $2.6092 \times 10^{-8}$ \\
\hline 12 & $16 \mathrm{M}_{\square}$ & $40.96 \times 10^{68}$ & $2.4462 \times 10^{-8}$ \\
\hline 13 & $17 \mathrm{M}_{\square}$ & $49.13 \times 10^{68}$ & $2.3023 \times 10^{-8}$ \\
\hline 14 & $18 \mathrm{M}_{\square}$ & $58.32 \times 10^{68}$ & $2.1744 \times 10^{-8}$ \\
\hline 15 & $19 \mathrm{M}_{\square}$ & $68.59 \times 10^{68}$ & $2.0599 \times 10^{-8}$ \\
\hline 16 & $20 \mathrm{M}_{\square}$ & $80.00 \times 10^{68}$ & $1.9569 \times 10^{-8}$ \\
\hline
\end{tabular}

Table 2. Life time \& Temperature of black holes in AGN.

\begin{tabular}{|c|c|c|c|c|c|c|}
\hline S.N. & $\begin{array}{l}\text { Mass of BHs } \\
\text { (M) }\end{array}$ & $\log _{10} \mathrm{M}$ & $\begin{array}{c}\text { Life time of } \\
\text { BHs }(\Gamma) \text { in year }\end{array}$ & $\log _{10} \Gamma$ & $\begin{array}{c}\text { Temp. of BHs (T) } \\
\text { in kelvin } \\
(\mathrm{G}=\mathrm{c}=\mathrm{h}=\mathrm{k}=1)\end{array}$ & $\log _{10} \mathrm{~T}$ \\
\hline 1 & $1 \times 10^{6}$ & 6.0000 & $1 \times 10^{84}$ & 84.0000 & $398.1000 \times 10^{-10}$ & -7.4000 \\
\hline 2 & $2 \times 10^{6}$ & 6.3010 & $8 \times 10^{84}$ & 84.9030 & $199.0500 \times 10^{-10}$ & -7.7010 \\
\hline 3 & $3 \times 10^{6}$ & 6.4771 & $27 \times 10^{84}$ & 85.4313 & $132.7000 \times 10^{-10}$ & -7.8771 \\
\hline 4 & $4 \times 10^{6}$ & 6.6020 & $64 \times 10^{84}$ & 85.8061 & $99.5250 \times 10^{-10}$ & -8.0020 \\
\hline 5 & $5 \times 10^{6}$ & 6.6989 & $125 \times 10^{84}$ & 86.0969 & $79.6200 \times 10^{-10}$ & -8.0989 \\
\hline 6 & $6 \times 10^{6}$ & 6.7781 & $216 \times 10^{84}$ & 86.3344 & $66.3500 \times 10^{-10}$ & -8.1781 \\
\hline 7 & $7 \times 10^{6}$ & 6.8450 & $343 \times 10^{84}$ & 86.5352 & $56.8714 \times 10^{-10}$ & -8.2451 \\
\hline 8 & $8 \times 10^{6}$ & 6.9030 & $512 \times 10^{84}$ & 86.7092 & $49.7625 \times 10^{-10}$ & -8.3030 \\
\hline 9 & $9 \times 10^{6}$ & 6.9542 & $729 \times 10^{84}$ & 86.8627 & $44.2333 \times 10^{-10}$ & -8.3542 \\
\hline 10 & $1 \times 10^{7}$ & 7.0000 & $1 \times 10^{87}$ & 87.0000 & $39.8100 \times 10^{-10}$ & -8.4000 \\
\hline 11 & $2 \times 10^{7}$ & 7.3010 & $8 \times 10^{87}$ & 87.9030 & $19.9050 \times 10^{-10}$ & -8.7010 \\
\hline 12 & $3 \times 10^{7}$ & 7.4771 & $27 \times 10^{87}$ & 88.4313 & $13.2700 \times 10^{-10}$ & -8.8771 \\
\hline 13 & $4 \times 10^{7}$ & 7.6020 & $64 \times 10^{87}$ & 88.8061 & $9.9525 \times 10^{-10}$ & -9.0020 \\
\hline 14 & $5 \times 10^{7}$ & 7.6989 & $125 \times 10^{87}$ & 89.0969 & $7.9620 \times 10^{-10}$ & -9.0989 \\
\hline 15 & $6 \times 10^{7}$ & 7.7781 & $216 \times 10^{87}$ & 89.3344 & $6.6350 \times 10^{-10}$ & -9.1781 \\
\hline 16 & $7 \times 10^{7}$ & 7.8450 & $343 \times 10^{87}$ & 89.5352 & $5.6871 \times 10^{-10}$ & -9.2451 \\
\hline 17 & $8 \times 10^{7}$ & 7.9030 & $512 \times 10^{87}$ & 89.7092 & $4.9762 \times 10^{-10}$ & -9.3030 \\
\hline 18 & $9 \times 10^{7}$ & 7.9542 & $729 \times 10^{87}$ & 89.8627 & $4.4233 \times 10^{-10}$ & -9.3542 \\
\hline 19 & $1 \times 10^{8}$ & 8.0000 & $1 \times 10^{90}$ & 90.0000 & $3.9810 \times 10^{-10}$ & -9.4000 \\
\hline 20 & $2 \times 10^{8}$ & 8.3010 & $8 \times 10^{90}$ & 90.9030 & $1.9905 \times 10^{-10}$ & -9.7010 \\
\hline 21 & $3 \times 10^{8}$ & 8.4771 & $27 \times 10^{90}$ & 91.4113 & $1.3270 \times 10^{-10}$ & -9.8771 \\
\hline 22 & $4 \times 10^{8}$ & 8.6020 & $64 \times 10^{90}$ & 91.8061 & $0.9952 \times 10^{-10}$ & -10.0020 \\
\hline 23 & $5 \times 10^{8}$ & 8.6989 & $125 \times 10^{90}$ & 92.0969 & $0.7962 \times 10^{-10}$ & -10.0989 \\
\hline 24 & $6 \times 10^{8}$ & 8.7781 & $216 \times 10^{90}$ & 92.3344 & $0.6635 \times 10^{-10}$ & -10.1781 \\
\hline 25 & $7 \times 10^{8}$ & 8.8450 & $343 \times 10^{90}$ & 92.5352 & $0.5687 \times 10^{-10}$ & -10.2451 \\
\hline 26 & $8 \times 10^{8}$ & 8.9030 & $512 \times 10^{90}$ & 92.7092 & $0.4976 \times 10^{-10}$ & -10.3030 \\
\hline 27 & $9 \times 10^{8}$ & 8.9542 & $729 \times 10^{90}$ & 92.8627 & $0.4423 \times 10^{-10}$ & -10.3542 \\
\hline 28 & $1 \times 10^{9}$ & 9.0000 & $1 \times 10^{93}$ & 93.0000 & $0.3981 \times 10^{-10}$ & -10.4000 \\
\hline 29 & $2 \times 10^{9}$ & 9.3010 & $8 \times 10^{93}$ & 93.9030 & $0.1990 \times 10^{-10}$ & -10.7010 \\
\hline 30 & $3 \times 10^{9}$ & 9.4771 & $27 \times 10^{93}$ & 94.4113 & $0.1327 \times 10^{-10}$ & -10.8771 \\
\hline 31 & $4 \times 10^{9}$ & 9.6020 & $64 \times 10^{93}$ & 94.8061 & $0.0995 \times 10^{-10}$ & -11.0020 \\
\hline 32 & $5 \times 10^{9}$ & 9.6989 & $125 \times 10^{93}$ & 95.0969 & $0.0796 \times 10^{-10}$ & -11.0989 \\
\hline
\end{tabular}




\section{Graph}

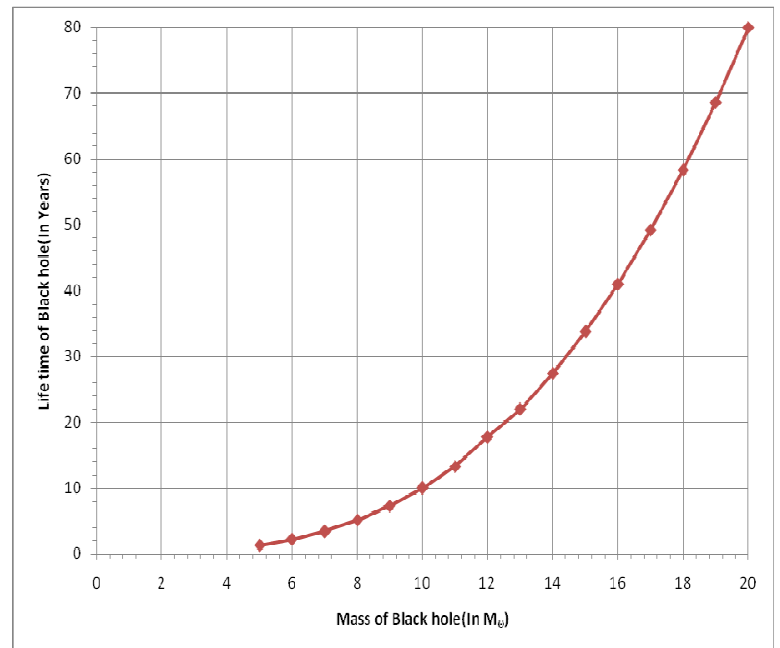

Fig 1. Graph plotted between mass of different test of black holes and corresponding life time in XRBs.

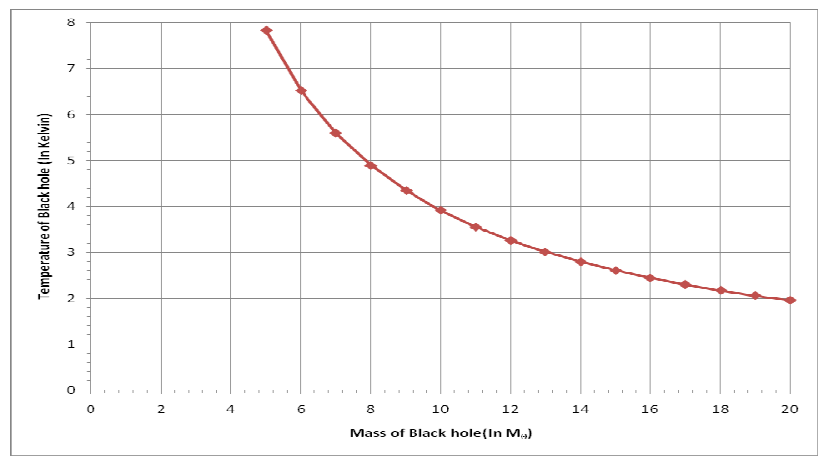

Fig 2. Graph plotted between mass of different test of black holes and corresponding temperature in XRBS.

\section{Results and Discussion}

The expressions for the lifetime and temperature of the black holes shows that the lifetime $(\Gamma)$ of the black hole is directly proportional to the cube of the mass of the black hole, and temperature ( $\mathrm{T}$ ) of the black hole is inversely proportional to the mass of the black hole. In the present article, we have calculated the lifetime and temperature of the black holes existing in $\mathrm{X}$-ray binaries and active galactic nuclei with the help of formula $\Gamma=\left(\mathrm{M} / \mathrm{M}_{\square}\right)^{3} \times 10^{66}$ years and $\mathrm{T}=\mathrm{hc}^{3} / 8 \pi \mathrm{kGM}$ Kelvin respectively. To analyze the lifetime and temperature of the black holes statistically, the graphs have been plotted between:-

(1) the mass of different test black holes and their corresponding life time in XRBs (fig. 1 ).

(2) the mass of different test black holes and their corresponding temperature in XRBs (fig. 2 ).

(3) the mass of different test black holes and their corresponding life time in AGN using logarithmic scale (fig. 3 ).

(4) the mass of different test black holes and their corresponding temperature in AGN using

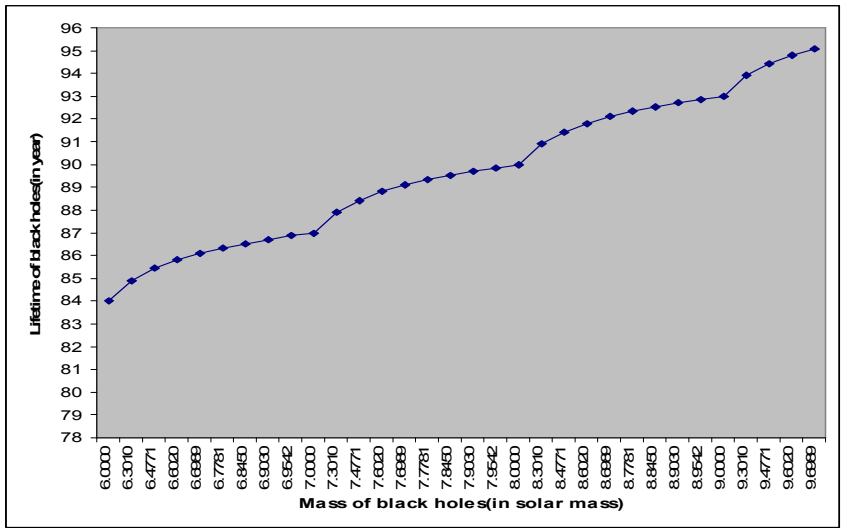

Fig 3. Graph plotted between mass of different test of black holes and corresponding life time in AGN using logarithmic scale.

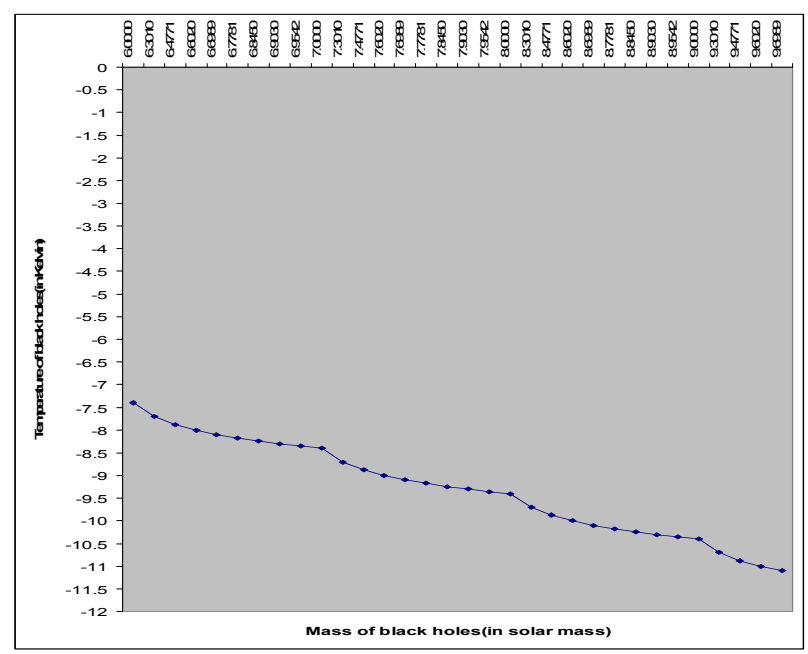

Fig 4. Graph plotted between mass of different test of black holes and corresponding temperature in AGN using logarithmic scale.

logarithmic scale (fig. 4 ).

The figure(1) shows that the life time of the black hole increases with the increase of the mass gradually and approximately linearly from $5-20 \mathrm{M}_{\square}$, while the figure (2) shows that the temperature of black hole decreases with increase of the mass gradually from 5-14 $M_{\odot}$ and approximately linearly from $15-20 \mathrm{M}_{\square}$ in XRBs.

The situation is quite different in the case of AGN. The figure(3) shows that the life time of the black hole increases with the increase of the mass gradually like transverse wave pattern from $10^{6}-10^{9.5} \mathrm{M}_{\square}$, while the figure (4) shows that the temperature of black hole decreases gradually with increase of the mass like transverse wave pattern from $10^{6}-10^{9.5} \mathrm{M}_{\square}$.

The reasons for differing in the nature of graph plotted between mass of different test black holes and life time \& temperature existing in XRBs and AGN are explained as follows: The black holes existing in AGN are super massive black holes and recent data indicates that the super massive black hole is more than 4 million solar masses ranging from $10^{6} M_{\odot}$ to $10^{9.5} M_{\odot}$. The difference in mass between two successive super massive black holes is roughly in order of 
$10 M_{\odot}$ giving the large values in the corresponding life time and temperature, whereas this difference for black holes existing in XRBs is simply in order of $1 M_{\odot}$ giving the normal values in the corresponding life time and temperature.

\section{Conclusion}

In the study of present research paper, we can draw the following conclusions.

(1) The life time of the black hole increases gradually with increase of the mass of different test black holes in XRBs, while like transverse wave pattern in AGN.

(2) The life time of the black hole decreases gradually with increase of the mass of different test black holes in XRBs, while like transverse wave pattern in AGN.

\section{Acknowledgement}

The authors are grateful to the reviewers and editor for pointing out the errors in the original manuscript and making constructive suggestions. The authors are obliged to Dr. Gopi Kant Jha, Former Head \& Prof. of Physics, L.N.M.U Darbhanga and Dr. Neeraj Pant, Associate Professor, Dept. of Mathematics, N.D.A. Khadakwasala, Pune for their inspiration and motivation.

\section{References}

[1] Mehta, V. K. and Mehta, R. (2008): "Principle of Physics", S. Chand \& Company.

[2] Wald, R. M. (2001): “The thermodynamics of black holes", Living reviews in relativity.

[3] Hawking, S.W.(1974): “Black hole explosion?” Nature.
[4] Charlie, R.: A conversation with Dr. Stephen Hawking \& Lucy Hawking.

[5] Kinoshita, S. and Tanahashi, N. (2012): "Hawking temperature for near-equilibrium black holes." arXiv:1111.2684v2 [hep-th].

[6] Triyanta \& Bowaire, A. N. (2013): "Hawking Temperature of the Reissner-Nordstrom-Vaidya Black Hole", J. Math. Fund. Sci., Vol. 45, No. 2, 114-123.

[7] Zee, A. (2003): "Quantum Field Theory in a Nutshell", Princeton University Press.

[8] Silva, P. R. (2006): "A note on black hole temperature and entropy." General Relativity and Quantum Cosmology, arXiv.org/pdf/gr-qc/0605051.

[9] Ram, B., Ram, A. and Ram, N.(2005): "The quantum black hole" arXiv:gr-qc/0504030v1.

[10] Narayan, R. (2005):"Black Holes in Astrophysics," New Journal of Physics, Vol.7, No.1, pp.1-31.

[11] Ferrarese, L. and Ford, H.(2005): "Super massive Black Holes in Galactic Nuclei: Past Present \& Future Research", Space Science Reviews 116..523-624, DOI: 10.1007/s11214-005-3947-6.

[12] Blandford, R. D.(1999): "Recent results on Active Galactic Nuclei." Astrophysics and Space Science 261:245-252.

[13] Ferrarese, L., Ford, H. C. and Jaffe, W. (1996): "Evidence for a Massive Black Hole in the Active Galaxy NGC 4261 from Hubble Space Telescope Images and Spectra," The Astrophysical Journal, Vol. 470,p. 444. doi:10.1086/177876 .

[14] Madejski, G.(2003): "Black Holes in Active Galactic Nuclei", SLAC-PUB-9702.

[15] Sargent, W.L.W. et al.(1978) :Dynamical Evidence for a Central Mass Concentration in the Galaxy M87, The Astrophysical Journal, Vol. 221, p.731.

[16] Mehta, R.N. et al.(2013): "Study of variation of temperature of black holes with respect to the mass in XRBs." International Journal Astrophysics and Space Science, doi: 10.11648/j.ijass.20130104. 